\title{
Some experiments on figural effects in binocular rivalry '
}

PAUL WHITTLE, ${ }^{2}$ D. C. BLOOR AND S. POCOCK

THE PSYCHOLOGICAL LABORATORY, CAMBRIDGE, ENGLAND

The four experiments in this paper were designed to investigate two questions. First, does a stimulus which is dominant in rivalry tend to make nearby stimuli seen by the same eye dominant also? The results suggest that it does not. Second, under what conditions do nearby stimuli, rivalling with opponents of equal strength, rival synchronously? The experiments show that contour-segments belonging to the same line, even when seen by different eyes, rival synchronously: Mere contiguity, however, does not lead to synchrony.

In trying to understand binocular rivalry, it is important to know to what extent the alternations in one region of the visual field are independent of those in another. The size and nature of the rivalling units might be expected to reveal something of the mechanisms of rivalry and perhaps of form perception in general, paralleling the suggestive studies of the fragmentation of the stablized image (e.g., Pritchard, Heron, \& Hebb, 1960). It is easy to verify Helmholtz's (1856) observation that "ordinarily, in the various parts of the field, one image will prevail more than the other, whereas in other parts the other image will predominate," but this aspect of rivalry has received surprisingly little attention.

In what follows "rivalry" will mean binocular rivalry between contours, or between forms with sharply defined edges. The ill-defined patchy rivalry such as can be seen when each eye is presented with large uniform fields of different colors, will not be considered.

Rivalry in one region of the field can be affected by that in another. One phenomenon which is easy to observe shows that such effects can extend over several degrees. In an experiment which will not be reported in detail, but for which the conditions were similar to those described later, congruent black and white circles $8 \mathrm{deg}$ in diameter were seen to rival as whole figures for $80 \%$ of the time (average for five Ss with four trials each). That is to say, for that time either one or the other circle was seen complete. Now it will be shown that under other conditions the rivalries in regions less than $1 \mathrm{deg}$ apart are independent. The experiments in this paper constitute an attempt to discover what processes kept the 8 deg circles intact.

First, it is possible that a contour which is dominant, momentarily or permanently, tends to makc a nearby one seen by the same eye dominant also. This would imply that a patchy mixture of two figures is a less stable state than the complete dominance of one or the other figure. Experiments 1 and 2 looked for independent evidence for this influence of a dominant contour on nearby contours. The strategy of both experiments was as follows. Rivalry was recorded between a pair of intersecting lines. More lines were then added to, say, the right eye's field, which were visible most of the time because they corresponded to uniform regions in the other eye. The question was, did these added lines make the original right eye line more dominant?

Both experiments gave mainly negative results. It seemed possible that this failure to find much link-up between the added lines and the original ones was due to the great difference in the strengths of the corresponding stimuli in the other eye: a uniform region and a line respectively. It could be that when nearby stimuli are rivalling with equally strong opponents, the rivalries tend to be synchronized, so that at one moment all the left eye stimuli are dominant and at another all the right. This indeed describes the behavior of the 8 deg black and white circles. In Experiments 3 and 4 we tried to determine some of the conditions for such synchrony. For example, do the "nearby stimuli" have to form part of the same line?

\section{EXPERIMENT 1. The Influence on Rivalry of a Neighboring Predominant Contour}

The display is shown in Fig. 1. The rivalry recorded was that between the diagonal lines at their intersection in the center of the display. The circle $A$ and its inscribed square helped to maintain constant vergence. The smaller circle, $B$, was seen by the right eye only and was visible for most of the time: an instance of the predominance in rivalry of contours over uniform areas. The purpose of this experiment was to find out whether this predominating stimulus would tend to make the diagonal seen by the same eye also predominant. Therefore, for comparison, the rivalry was recorded also with displays identical to those in Fig. 1 except that they both lacked the small Circle B. The experiment was thus an attempt to see whether the rivalry at one point was independent of that at another in the extreme case where the second rivalry was almost complete predominance.

The size of Circle B, 1 deg in diameter, was chosen after preliminary experiments had shown that a circle $6 \mathrm{deg}$ in diameter had no detectable effect on the rivalry at its center.

\section{Method}

The following details apply to all the experiments in this paper. Stimulus materials and apparatus. The displays were drawn in matt black ink on white cardboard, illuminated with tungsten bulbs, and seen in a stereoscope using front surface mirrors. The displays were at an optical distance of 26 in. The lines used in Experiments 1 through 3 were about $5 \mathrm{~min}$ of arc wide. In Experiment 4 they were only about $2 \mathrm{~min}$ wide. The luminances of the black and white parts of the field were approximately 1.90 and $0.80 \log \mathrm{ft}-\mathrm{L}$ respectively. The visual field was black outside a circle of $16 \mathrm{deg}$ diameter in Experiments 1 and 2, or $10 \mathrm{deg}$ in Experiments 3 and 4.

Procedure. The displays were adjusted for each individual so as to be correctly superimposed in the binocular field. The Ss were told to stop recording if the monocular displays appeared to move apart (due to vergence changes) during a trial. If they did not return soon, they were realigned. Ss were always given as much practice as they wanted in order to feel confident they were recording the rivalry as accurately as they could. This was usually between two and four 1-min trials. They recorded the rivalry by holding down microswitches operating electrical stop-clocks cumulating the time for which the switches were pressed. Counters recorded the number of presses. Although we analyzed these counts in some detail, they did not add much to our understanding of the results. This data will therefore be only briefly reported.

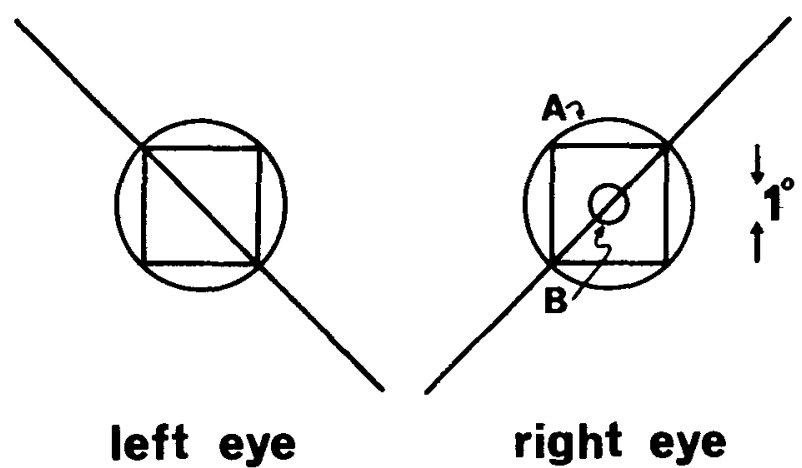

Fig. 1. Displays for Experiment 1. 
Trials comprised $1 \mathrm{~min}$ of recording (45 sec in Experiment 3) started after the $S$ had pressed the switch two or three times. Intertrial rests were about $1 \mathrm{~min}$ long. Trials with different conditions were randomly intermixed, unless otherwise stated.

Subjects. The Ss were students or young graduate staff in this University. About a third of them had previous experience in perceptual experiments.

Procedure specific to Experiment 1. The Ss were asked to press one of two microswitches according as to which diagonal was continuous across the point of intersection. Even though both lines were sometimes seen together at the intersection, one or the other always dominated, that is, appeared clearer than, darker than, or in front of the other. There were $11 \mathrm{Ss}$. They were asked to fixate as accurately as possible the point of intersection of the diagonals. There were eight trials with Circle B and eight without, randomly intermixed.

\section{Results}

The small circle did have a slight tendency to make the associated diagonal more dominant. Table 1 shows for each $S$ the predominance " $\mathrm{P}$ " of the diagonal seen by the right eye over that seen by the left. $P$ is the ratio of the average total amounts of time in each trial for which each diagonal was seen (the average being taken over the eight trials with the same displays). P tended to be greater when the circle B was present in the right eye's display than when it was absent $(p=0.048$, one-tailed, by the Walsh Test applied to Log P; Siegel, 1956). The average duration of single periods of dominance of the right eye diagonal was $1.9 \mathrm{sec}$ without Circle $\mathrm{B}$, and $2.4 \sec$ with $B$.

\section{A Supplementary Experiment}

We have assumed up to now that the small circle was visible for all or most of the time. However, a few Ss reported that it did sometimes disappear completely. An obvious question arose: were the Ss for whom Circle B made its diagonal more dominant, also those for whom Circle B itself was most dominant? With this in mind, the same 11 Ss were given five further $1-\mathrm{min}$ trials, in which they were asked to record only the amount of time for which the small circle completely disappeared. The average disappearance times, as percentages of the trial length, are also given in Table 1 and show an unexpected result: there is a weak positive correlation between the time for which Circle B disappeared and the ratio ( $P$ with Circle $B) /(P$ without Circle $B)$. That is, the less the circle was visible, the more its diagonal predominated. (Spearman's Rank Correlation Coefficient $=.565, \mathrm{p}<0.10$, twotailed. In Table 1 the Ss are arranged in rank order of the above ratio. S 1 is clearly an exception, but the only striking one, to this correlation.)

\section{Discussion}

First, it is remarkable that when the circle is at least partly dominant for $90 \%$ of the time (Ss 5 to 11 ) it has virtually no effect on the rivalry at its center, only $30 \mathrm{~min}$ of arc away from the domiinant contourx Second, the supplementary experiment shows that the more predominant Circle B is, the less tendency it has to make its associated diagonal predominate. Thus it seems likely that Circle B increased the dominance of the associated diagonal only if it rivalled to some extent synchronously with the diagonal, so that the two formed, as it were, a single compound object. The compound object may have predominated because it had three separate lines intersecting the single line seen by the other eye. This would agree with Breese (1899) who found that in the rivalry of red and green squares with diagonal lines drawn on them, squares with more lines predominated more. We can therefore not take the main result of the experiment-the apparent slight tendency of Circle B to make its diagonal more predominant-at its face value. The result of the supplementary experiment suggests that the influence of a contour $X$ on the rivalry of a nearby contour $Y$ seen by the same eye, depends primarily not on the predominance of $X$, but on the extent to which $X$ and $Y$ rival synchronously. Before studying further this postulated synchronous rivalry, another possibility must be considered. It may be
Table 1

Results of Experiment 1*

\begin{tabular}{cccc} 
Subject & $\begin{array}{c}\text { P with } \\
\text { circle }\end{array}$ & $\begin{array}{c}\text { P without } \\
\text { circle }\end{array}$ & $\begin{array}{r}\text { Per cent time } \\
\text { circle invisible }\end{array}$ \\
\hline 1 & 1.66 & 0.88 & 0.6 \\
2 & 1.02 & 0.55 & 26.5 \\
3 & 0.65 & 0.42 & 27.8 \\
4 & 1.06 & 0.73 & 10.6 \\
5 & 1.27 & 1.06 & 1.8 \\
6 & 1.09 & 0.91 & 5.7 \\
7 & 1.26 & 1.10 & 6.5 \\
8 & 1.21 & 1.18 & 2.6 \\
9 & 0.98 & 1.03 & 0.0 \\
10 & 1.00 & 1.08 & 0.0 \\
11 & 2.50 & 3.11 & 0.9 \\
\hline Average & 1.19 & 0.96 & 4.5 \\
\hline
\end{tabular}

*P is the ratio of the times for which the diagonals seen by the right and left eyes were dominant. The average Ps are geometric means.

that the tendency looked for in the previous experiment for a dominant contour segment to pull a neighboring one into dominance with it, is stronger if the two form part of the same smooth continuous line. The outlines of the circles whose rivalry-as-wholes partly stimulated this enquiry, did form such a continuous line, but the diagonal and the small circle $B$ in Experiment 1, which met at an abrupt right angle, did not.

Such a tendency would clearly be akin to the closure reported by Hempstead (1900): the filling-in of gaps in dimly perceived figures. (Though Hempstead found as many, if not more, instances of disintegration and complication in his S's reports, as of closure.)

\section{EXPERIMENT 2: Closure in Rivalry}

The displays are shown in Fig. 2. The left eye display was always a horizontal line, while the right eye one was either (a) a small arc of a circle, or (b) a complete circle containing the arc (a). This circle intersected the line at only one point. Most of the circle was visible for most of the time, since it corresponded to a uniform region in the other field. If the arc predominated more in (b) than in (a), this could be described as closure. The square frame helped to maintain constant vergence.
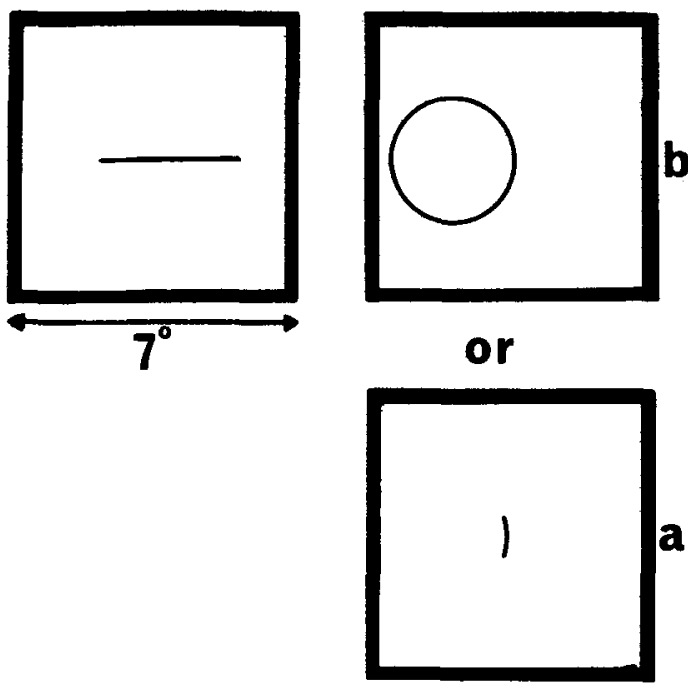

\section{left eye right eye}

Fig. 2. Displays for Experiment 2. 


\section{Procedure and Results}

Ss were asked to fixate the point of intersection of the line and the arc, and to press one of two microswitches according as to which stimulus was dominant at the point of intersection. Nine Ss were given eight trials with each display, randomly intermingled.

The predominances, $\mathrm{P}$, of the circle and the arc over the line are shown in Table 2. There is little difference between the results for the two displays. The slight tendency for the arc to predominate more when isolated than when forming part of the circle-the opposite of closure-does not reach statistical significance (twotailed $p>.10$, Wilcoxon Test on Log P). The average duration of individual periods of dominance was $3.0 \mathrm{sec}$ both for the circle and for the arc.

\section{Discussion}

This result is consistent with our interpretation of Experiment 1. In that experiment there was reason to attribute what positive effect was found to the synchronous rivalry of the diagonal and the small circle, predominating because they formed three lines intersecting the single left eye line. But in this case the circle, even if it rivalled as a single object, which is unlikely, would not have the same reason to be more dominant than the arc alone, since there is only a single line in each eye. The results of the two experiments thus agree; there does not seem to be a general tendency for a dominant contour-segment to increase the dominance of a nearby one seen by the same eye.

EXPERIMENT 3: Are Nearby Similar Rivalries Synchronous?

When two similar neighboring contour-segments seen by the same eye are each rivalling with similar contour-segments, are the two rivalries synchronous? That is, do the two left eye contours appear and disappear together, and therefore the right eye ones also? If this can be shown to be generally true, then we can regard the rivalry-as-wholes of the $8 \mathrm{deg}$ circles as an instance of it.

Experiment 3 was an attempt to answer this question. using the displays shown in Fig. 3. The $\mathbf{S}$ saw two pairs of rivalling stimuli, each consisting of a vertical dash rivalling with a horizontal dash. The pairs were one above the other, so that the two vertical dashes were in line. In some trials Ss were asked to press a switch when one particular dash was completely visible, ignoring its partner and the other pairs. This was done for each of the four dashes. In other trials the S pressed a switch when a particular pair of dashes, one from each of the rivalling pairs, was completely visible. For example, the pair forming an upright T ("UT"), or the two vertical dashes (" $2 \mathrm{~V}$ "). (The other pairs will be denoted "IT," inverted $\mathrm{T}$; " $2 \mathrm{H}$," two horizontal dashes; the single dashes by "UV," "UH," "LV" and " $L H$ " for upper vertical dash, etc.)

Now if the rivalry of the upper pair of dashes is independent of the lower, in the sense that the two are not synchronous, then the probability of seeing a particular pair of dashes at any moment is the product of the probabilities of seeing each of the components. Let us call the percentage of viewing time for which each object is seen $O$ (UV), $O(2 \mathrm{H})$ etc., and the percentage of time for which we would expect to see any pair of dashes, on the hypothesis of independence, $E(2 \mathrm{H})$ etc. (" $\mathrm{O}$ " for observed and " $E$ " for

Table2

Results of Experiment 2*

\begin{tabular}{ccc} 
Subject & P for circle & P for arc \\
\hline 1 & 2.07 & 1.72 \\
2 & 1.41 & 1.62 \\
3 & 1.16 & 1.78 \\
4 & 0.64 & 0.70 \\
5 & 1.10 & 1.22 \\
6 & 1.32 & 1.46 \\
7 & 1.06 & 1.28 \\
8 & 1.82 & 1.89 \\
9 & 1.92 & 1.84 \\
\hline Geometric means & 1.32 & 1.45 \\
\hline
\end{tabular}

* $P$ is the ratio of the times for which the arc (or the circle) seen by the right eye, and the horizontal line seen by the left eye were dominant.

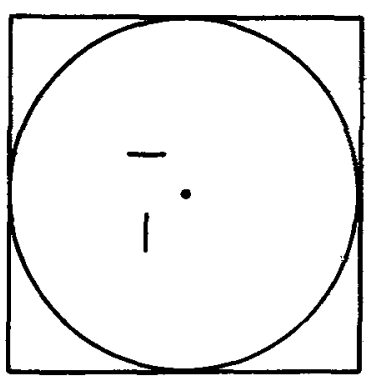

a

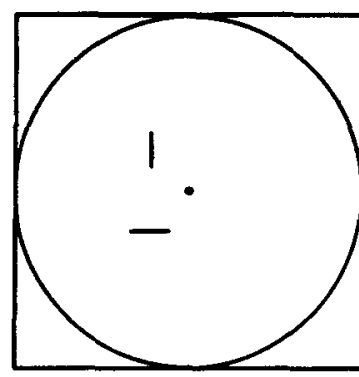

$\left[1^{\circ}\right.$ scale
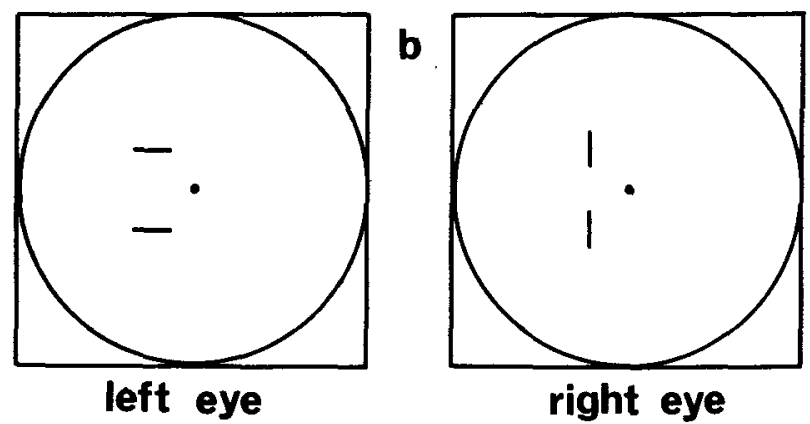

Fig. 3. Displays for Experiment 3.

expected). Now the probability of seeing the upper vertical dash, say, at any moment in the rivalry is $\mathrm{O}$ (UV)/100. Therefore we have four relationships of the form.

$\mathrm{E}(\mathrm{IT})=100 \times \mathrm{O}$ (UV) $\times \mathrm{O}(\mathrm{LH})$. The extents to which $\mathrm{O}$ exceeds $E$ for each of the pairs IT, $2 \mathrm{H}$, etc. provide a measure and a description of the synchrony of the two rivalling pairs.

Comparison of the results with displays (a) and (b) should tell us whether the synchrony links dashes seen by the same eye, or dashes which are parts of the same line-the two vertical dashes-or whether both tendencies exist.

The size of the dashes was chosen so that they would usually appear and disappear as wholes. That is, each dash tends to be either present and complete, or wholly absent. This makes the observers' task easier. The distance apart was chosen with regard to the radius of action of rivalry, so that the stimuli of the upper pair would not rival with the lower ones. This was checked in some extra trials with different displays (c) and (d). Display (c) comprised the upper vertical dash to the left eye and the lower vertical dash to the right. There were no horizontal dashes. (d) was like (c) except that the dash to the right eye was omitted. (d) allows us to measure Troxler's Effect (the disappearance of peripherally viewed objects, during steady fixation), and thus to see whether such disappearances as occur with (c) are rivalry or simply Troxler's Effect.

\section{Procedure}

The $\mathbf{S}$ was given only one microswitch, and his instructions were: "Press the switch when and only when the upper horizontal dash is dominant," or ". . . when an upright T is visible," etc. They were asked to fixate the small black dot to the right of the dashes. For (a) and (b) two trials were given for observing each single dash; i.e., eight such trials with (a) and another eight with (b). Four trials were given for each pairwise combination. Two trials with each of (c) and (d) were given, the $S$ recording the disappearances of the upper dash. All trials lasted $45 \mathrm{sec}$ with rests of about $30 \mathrm{sec}$ in between. There were eight Ss, each of whom came for two sessions of about $1 \mathrm{~h}$ each.

\section{Results}

The percentages of time for which the various dashes and pairs of dashes were seen are given in Table 3, averaged over all Ss. The 
Table 3 Results of Experiment 3*

(a) UT v IT

(b) $2 \mathrm{~V}$ v $2 \mathrm{H}$

\begin{tabular}{cccr} 
& & (a) UT V IT & (b) 2 V v 2H \\
\hline Upper & UV & $36.0(10.7)$ & $33.7(9.1)$ \\
pair & UH & $37.7(13.4)$ & $37.8(6.8)$ \\
Lower & LV & $31.3(11.2)$ & $38.5(9.2)$ \\
pair & LH & $45.5(18.8)$ & $33.6(8.9)$ \\
& O & $17.0(7.4)$ & $26.6(6.3)$ \\
2V & E & $11.7(6.5)$ & $13.8(4.1)$ \\
& O- E & $+5.3(5.0)$ & $+12.8(4.0)$ \\
& O & $19.8(7.5)$ & $22.8(6.5)$ \\
2H & E & $18.4(11.0)$ & $12.7(4.0)$ \\
& O-E & $+1.4(11.1)$ & $+10.0(5.0)$ \\
& O & $18.6(8.8)$ & $9.7(4.5)$ \\
IT & E & $18.0(10.8)$ & $11.6(4.1)$ \\
& O-E & $+0.6(4.7)$ & $-1.8(1.4)$ \\
& O & $13.5(5.9)$ & $12.8(5.9)$ \\
UT & E & $12.4(7.7)$ & $14.7(4.0)$ \\
& O-E & $+1.1(4.8)$ & $-1.8(4.9)$ \\
\hline
\end{tabular}

*Average percentages of viewing time for which each single dash (top four rows of table), and the various pairs (rows labelled $O$ ) were seen. Figures in parentheses are standard deviations. Rows labelled $E$ give the calculated per cent viewing time for each pair on the hypothesis that the upper and lower rivabies are independent. The left-hand column is avenages over 10 subjects, the right-hand column over eight.

most important figures are those for (O-E), the amount by which the percentage time for which any pair of dashes was seen exceeded the expectation on the hypothesis of independent upper and lower rivalries.

For display (a) (O-E) is significantly $>0$ only for the pair of vertical dashes (two-tailed $p=.01$, Wilcoxon Test). But this result must be interpreted with caution, since the differences in (O-E) over the four different pairs are barely significant $(.05<p<.10$, Friedman Two-way Analysis of Variance, Siegel, 1956). Of course this is not a conflict; it is due to the fact that while the pair of vertical dashes are synchronous for all Ss, for some Ss other pairs are more so. Perhaps the most striking finding with display (a) is that for neither IT nor UT, the right and left eye pairs, is (O-E) significantly different from zero.

This is in marked contrast to display (b) where for both $2 \mathrm{~V}$ and $2 \mathrm{H}$, which are the right and left eye pairs in this display, (O-E) was positive for all Ss (so two-tailed $p=.008$, Binomial Test). Here the Friedman Analysis of Variance showed that there were consistent differences in $(O-E)$ over the four pairs $(p<.001)$. Again $(O-E)$ for IT and UT was not significantly different from zero.

Thus there was synchrony between the vertical dashes in both displays, but more so when they were present to the same eye. ((O-E) for $2 \mathrm{~V}$ was greater for (b) than for (a) with a one-tailed $p=.03$ by the Wilcoxon Test. For $2 \mathrm{H}, \mathrm{p}=.06$. For IT and UT (O-E) did tend to be greater for (a) than for (b), with $\mathrm{p}=.21$ and .07 respectively.)

On the other hand there was synchrony between the stimuli present to the same eye only when those stimuli formed a line (2V) or the complementary pair (2H). (Statistical details above, and also it was true that for both eyes of all eight $S$ s the synchrony between pairs to the same eye was greater with (b) than with (a).)

The average durations of single periods of dominance were 1.9 sec for single dashes and $1.3 \mathrm{sec}$ for pairs. (A difference in this direction was shown by all Ss with both displays.) For display (a) the greater synchrony of $2 \mathrm{~V}$ was associated not with longer periods of dominance but with there being more of them. With displays (b) on the other hand the greater synchrony of $2 \mathrm{~V}$ and $2 \mathrm{H}$ was reflected both in longer periods of dominance ( $1.5 \mathrm{sec}$ for these pairs, compared with $1.1 \mathrm{sec}$ for the others) and in their more frequent occurrence.

The results with displays (c) and (d) showed that there was no rivalry between the upper and lower vertical dashes. The upper vertical dash disappeared for $13.9 \%$ of the time when there was no lower dash (display (d)), and for $14.1 \%$ when there was (display (c)). The correlation between Ss' scores with the two displays was +.98 (Spearman's Rank Correlation Coefficient). The results with both displays can therefore be attributed to Troxler's Effect. The same result would be expected for other dash-pairs.

\section{Discussion}

There is thus good evidence that contour segments which are in line are seen together in rivalry more often than would be expected by chance. At first sight the fact that this synchrony is stronger when they are seen by the same eye suggests that there is an additional tendency for stimuli seen by the same eye to be synchronous. However, there is only the weakest indication that this intra-eye linkage exists at all for pairs of stimuli which do not form a line (or the complementary pair $2 \mathrm{H}$ ). Thus it would seem more parsimonious to suppose that the linkage between dashes in line is the only one present, and that this is weaker with display (a) because eye-movements will cause them to be less accurately aligned. It would not be worth pressing this interpretation at present since we would expect to find an intra-eye linkage either if the displays were made smaller, or if they were further from the fixation point. Further, there is a suggestion of an intra-eye linkage in the results for some Ss with display (a).

It can be seen from Table 3 that the percentage times for which each dash of a rivalling pair was seen add up to about 75 rather than $100 \%$. Ss' reports indicated that this was largely because it was not clear which dash was dominant, for about $25 \%$ of the time. (Unlike the foveal rivalry of intersecting contours in Experiments 1 and 2.)

Can we be sure that the synchrony found was due to rivalry rather than Troxler's Effect? The result with displays (c) and (d), showing that each dash disappears on average for $14 \%$ of the time even without a rivalling stimulus, suggests that complete synchrony of these Troxler disappearances, without any synchrony in rivalry, could account for the results. This possibility can be discounted because there was no correlation between the extent of Troxler's Effect and the amount of synchrony shown, over the eight Ss. (Spearman's Rank Correlation Coefficient $=.21$.) It would of course be of interest to investigate the amount of synchrony shown in Troxler's Effect.

The rationale of this experiment assumes that the rivalry is independent of the S's set. For example, that he sees the upper vertical dash dominant for the same time when he is asked to record just that, as when he is asked to record the presence of the two vertical dashes. This is unlikely to be precisely true. A positive effect of attention to a stimulus on its dominance in rivalry has of ten been claimed (Helmholtz, 1856; Fry, 1936). In another experiment we asked eight $S$ s to record rivalry between contours intersecting in the fovea, like the display of Experiment 1 without the circle $\mathrm{B}$, under two different instructions. In eight trials they were given one switch to record the presence of just one contour ("A," say), and in another eight two switches, each to be pressed when the corresponding contour dominated. Five Ss consistently reported Contour A more in the first condition than the second, one showed the reverse effect, and two no consistent difference. The effect of instructions is thus not consistent over Ss, and there are two further reasons for believing that it did not seriously affect the present results. First, the very close agreement with the chance expectations of the times for which the UT and IT pairs were seen. Scond, there is no reason why the Ss' set should affect the perception of $2 \mathrm{~V}$ and $2 \mathrm{H}$ more than that of the other pairs. This discussion is closely related to the important question of whether the synchrony is due to perceptual or response mechanisms. Again there seems no reason why a response bias should favor one pair rather than another.

Diaz Caneja (quoted by Le Grand, 1956, p. 205) has claimed that segments of the same line, although presented to different eyes, tend to appear together in rivalry. We have not found his demonstrations convincing to all Ss, but the results of the present experiment do support his claim. The linkage of the two vertical lines seems likely to be connected with the locking of vergence movements which occurs with haploscopic displays used to 
Table 4

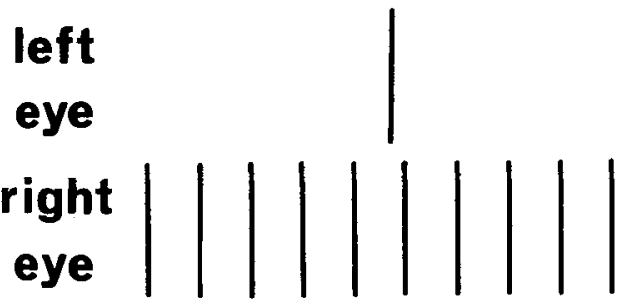

Fig. 4.

measure phoria, like that shown in Fig. 4. The upper line tends to be perceived as fairly exactly aligned with one of the lines in the lower row, rather than appearing above a gap.

EXPERIMENT 4: A Further Look at Rivalry-as-whole Figures

The $8 \mathrm{deg}$ circles mentioned above, which rivalled as wholes, were filled-in rather than outline figures, and their contours were continuous. In an experiment that will not be described in detail we investigated the rivalry of a circle, $8 \mathrm{deg}$ in diameter, composed of eight short arcs (like the arc in Experiment 2) with gaps between them, with a pattern of eight rays in the other eye. We found that the eight arcs were visible together much more than would be expected by chance. However, in later control experiments we found that the arcs disappeared almost as much when there was a blank field in the other eye as when the ray pattern was present. It was therefore impossible to say whether the results were due to Troxler's Effect or to rivalry. The experiment was therefore repeated with a simpler display using a circle only 48 $\mathrm{min}$ in diameter, its center being the fixation point. This display is shown in Fig. 5.

\section{Procedure and Results}

Exactly the same display was used throughout. The outer rings are to maintain constant vergence. Ss recorded the visibility of each single arc (two trials each); of each opposing pair (left eye pair and right eye pair, four trials each), and of all four arcs together (eight trials).

The average results for the nine Ss are given in Table 4. There is synchrony within each eye but none between eyes. The times for which the pairs of arcs would be expected to be visible together on the hypothesis that each arc rivals independently with its corresponding line, can be calculated as for Experiment 3, from the product of the probabilities of seeing each arc separately. These expectations are shown in parentheses in Table 4. For both left and right eyes the observed values are greater than the expected (one-tailed $p=.02$ and .03 respectively, on Wilcoxon Tests). The expected value shown for the visibility of all four arcs together was obtained by multiplying the probabilities that the left and right eye pairs should each be separately visible. That is, it represents the expected value on the hypothesis that the left and right eye pairs rival independently; not that all four arcs rival independently. The average durations of single periods of dominance were $5.9 \mathrm{sec}$ for single arcs, $4.1 \mathrm{sec}$ for pairs, and $2.5 \mathrm{sec}$ for all four arcs.

\section{Discussion}

This experiment was conceived and has been described in terms of the circle of dashes. But in the light of the results of Experiment 3 the results seem more likely to be due to synchrony between the opposing stimuli, which were portions of continuous vertical and horizontal lines. The results could be influenced by rivalry between the left eye arcs and the right eye arcs, or between the left and right eye lines, as opposed to rivalry of arcs with lines. To check on this we carried out some control experiments with eight new Ss in which either the lines or the arcs were omitted, and Ss recorded such rivalry as occurred between the arcs or lines respectively. These experiments showed that there was a small
Results of Experiment $4^{*}$

\begin{tabular}{lcccccc} 
& \multicolumn{2}{c}{ Left eye } & & \multicolumn{2}{c}{ Right eye } \\
\cline { 2 - 4 } & Upper & & Lower & & Right & Left \\
\hline Single ares & 64 & & 71 & & 74 & 69 \\
\hline Pairs of arcs & & $60(53)$ & & & $64(49)$ \\
\hline All four arcs & & & & $41(42)$ & & \\
\hline
\end{tabular}

*Figures are percentages of total viewing time for which the stated parts of the display were visible. Figures in, parentheses are pencentages expected on the hypothesis of independent rivalries.

amount of rivalry between the arcs and a little more between the lines: each pair of arcs was visible for $95 \%$ of the time and each line for $90 \%$, on average. It seems unlikely that such rivalry could have appreciably influenced the results. In further trials the amount of disappearance due to Troxler's Effect was shown to be negligible ( $2 \%$ of viewing time).

This experiment, then provides no evidence that the four arcs' forming a "good figure"-a circle-makes them appear together more often than would be expected by chance. Such synchrony as does occur, which is entirely intra-eye, may be attributable to the tendency, in Experiment 3, of segments of the same straight line to appear together.

\section{CONCLUSIONS}

In the introduction we suggested two possible processes which might underlie the observation that, under some conditions, quite large figures appear and disappear as wholes in binocular rivalry. The first was that a dominant contour may tend to increase the dominance of a neighboring contour seen by the same eye. The second was that nearby stimuli seen by the same eye may always tend to rival synchronously if they are competing with opponents of equal strengths. We have found very little other evidence for the first suggestion. We have shown that the second suggestion is not generally true. The rivalries of neighboring stimuli are synchronous only if they do form certain types of figure. In particular, there is synchrony between contour-segments which form a line, even when they are seen by different eyes.

Many other experiments remain to be done. In particular it would be of interest to see whether contour-segments are synchronous when they form parts of a curved or jagged but continuous line. Such synchrony as was obtained in Experiment 1 suggests that they would be. Mere contiguity does not produce synchrony. This was shown strikingly by the results for the upright and inverted $T$ combinations in Experiment 3 . When the conditions for synchrony do not obtain, rivalry can be a very local process. It depends on the properties of the stimuli within small cortesponding regions of the two retinae, but can be remarkably independent of stimuli less than one degree away. This argues against the hypothesis that changes in .rivalry are produced by factors such as eye-movements which affect the whole visual field.
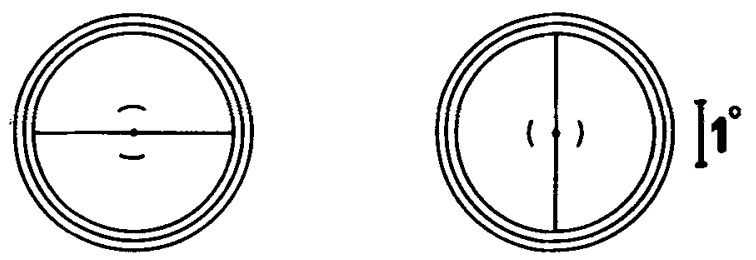

left eye

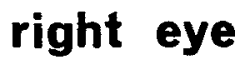

Fig. 5. Display for Experiment 4. 
These conclusions apply only to foveal and parafoveal rivalry, under steady fixation. When fixation is not controlled, the eyes are free to follow the contours of a figure present to one eye, and so keep the figure dominant in rivalry. This effect could have contributed to many of the reports in the literature of effects of the meaning and the emotional significance of stimuli on binocular rivalry (see Ittelson, 1962, for references).

It is difficult to relate our experiments to previous studies of the fragmentation of images, either in rivalry (Smith, 1968) or in stabilized (Pritchard et al, 1960) or after-images (Smith, 1968; Evans, 1966), because of differences in the aims and designs of the various experiments. In most previous studies the spontaneous fragmentations of connected figures have been observed. In our experiments, on the other hand, particular types of fragmentations were imposed on the figures; in Experiments 1 and 2 by the fact that only a small part of the figure intersected a rival stimulus; in Experiments 3 and 4 by deliberately constructing the figure out of discrete units. This method permits a more detailed quantitative analysis than is easy to apply to spontaneous fragmentations, and has revealed something of the figural processes determining what is seen in rivalry. In that our studies also suggest the existence of perceptual units corresponding to straight lines, or continuous lines, they are in general agreement with the previous work. However, the behavior of figures in rivalry is undoubtedly different from their behavior as stabilized or after-images. Smith (1968) showed that the spontaneous fragmentations of a figure in rivalry with "visual noise" appeared random by comparison with the behavior of the same figure as an after-image. In the latter condition fragmentations were more "structured," that is, lines acted as units, breaking off cleanly at corners. It would be of interest to conduct experiments on after-images of figures composed of discrete units, like Fig. 3 , using the more quantitative analysis possible, to gain more insight into the fragmentations of after-images and their relations to fragmentations in rivalry.

\section{REFERENCES}

BREESE, B. B. On inhibition. PsychoL Monogr., 1899, 3, No. 1.

EVANS, C. R. New approach to pattern perception. Discovery, 1966, 27, 17-21.

FRY, G. A. The relationship of accommodation to the suppression of vision in one eye. Amer. J. OphthaL, 1936, 19, 135-138.

HELMHOLTZ, H. von Handbuch der physiologischen Optik, 1856-1866. English translation 1924, reprinted. New York: Dover, 1962.

HEMPSTEAD, L. The perception of visual form. Amer. J. Psychol, 1900, 12, 185-192.

ITTELSON, W. A. Perception and transactional psychology. In S. Koch (Ed.), Psychology: A study of a science. Vol. 4. New York: McGraw-Hill, 1962. Pp. 660-704.

Le GRAND, Y. Optique physiologique. Vol. 3. Paris: Revue d'Optique, 1956.

PRITCHARD, R. M., HERON, W., \& HEBB, D. O. Visual perception approached by the method of stabilised images. Canad. J. Psychol, $1960,14,67-77$.

SIEGEL, S. Nonparametric statistics. New York: McGraw-Hill, 1956.

SMITH, P. K. Image fragmentations with after-images and in binocular rivalry. Psychon. Sci., 1968, 10, 275-276.

\section{NOTES}

1. We are grateful to Professor O. L. Zangwill for providing facilities for these experiments; to Mr. G. C. Grindley for encouragement and advice, and to Mrs. J. E. Latto for her part in some of the experiments. Experiments 1, 2 and part of 3 were carried out by P.W.; 1 and 2 while he held a scholarship from the Medical Research Council. Experiments 4 (D. C. B.) and part of 3 (S. P.) were undergraduate research projects.

2. Address: The Psychological Laboratory, University of Cambridge, Downing Street, Cambridge, England.

(Accepted for publication April 29, 1968.) 